Research Article

\title{
Biological Activities and Chemistry of Triterpene Saponins from Medicago Species: An Update Review
}

\author{
Guanzhen Wang $\left(\mathbb{D},{ }^{1}\right.$ Junlong Wang, ${ }^{1}$ Wei Liu $\left(\mathbb{D},{ }^{1}\right.$ Muhammad Farrukh Nisar $\left(\mathbb{D},{ }^{2,3,4}\right.$ \\ Mohamed A. El-Esawi $\mathbb{D}^{5},{ }^{5}$ and Chunpeng Wan $\mathbb{D D}^{2}$ \\ ${ }^{1}$ Key Lab of Natural Product Chemistry and Application at Universities of Education Department of Xinjiang Uygur Autonomous \\ Region, Yili Normal University, Yining 835000, China \\ ${ }^{2}$ College of Agronomy, Jiangxi Agricultural University, \\ Jiangxi Key Laboratory for Post-harvest Technology and Nondestructive Testing of Fruits \& Vegetables/Collaborative Innovation \\ Center of Post-harvest Key Technology and Quality Safety of Fruits and Vegetables in Jiangxi Province, Nanchang 330045, China \\ ${ }^{3}$ Key Laboratory of Crop Physiology, Ecology and Genetic Breeding, Ministry of Education, Jiangxi Agricultural University, \\ Nanchang, Jiangxi, China \\ ${ }^{4}$ Department of Physiology and Biochemistry, Cholistan University of Veterinary and Animal Sciences (CUVAS), \\ Bahawalpur 63100, Pakistan \\ ${ }^{5}$ Botany Department, Faculty of Science, Tanta University, Tanta 31527, Egypt
}

Correspondence should be addressed to Wei Liu; nculiuwei@126.com and Muhammad Farrukh Nisar; farrukh.nisar@ hotmail.com

Received 12 January 2021; Accepted 19 May 2021; Published 26 May 2021

Academic Editor: Juntra Karbwang

Copyright (c) 2021 Guanzhen Wang et al. This is an open access article distributed under the Creative Commons Attribution License, which permits unrestricted use, distribution, and reproduction in any medium, provided the original work is properly cited.

\begin{abstract}
Plants are known to be a great source of phytochemicals for centuries. Medicago, belonging to the Family Fabaceae, is a large and well spread genus comprising about 83 cosmopolitan species, of which one-third are annuals and span diverse ecological niches. Medicago species are rich in saponins mainly classified into three classes, namely, steroid alkaloid glycosides, triterpene glycosides, and steroid glycosides. These saponins are important compounds having diverse pharmacological and biological activities. As a whole, 95 of saponins are reported to date occurring in Medicago species using various latest extraction/isolation techniques. Considering the multiple biological and pharmacological potential of Medicago species due to saponins along with structural diversity, we compiled this review article to sum up the recent reports for the pharmacological potential of the Medicago's derived saponins in modern as well as traditional medication systems. The current manuscript produces data of chemical structures and molecular masses of all Medicago species saponins simultaneously. The toxicity of certain pure saponins (aglycones) has been reported in vitro; hederagenin appeared highly toxic in comparison to medicagenic acid and bayogenin against X. index, while soyasaponin I, containing soyasapogenol B as a glycone, appeared as the least toxic saponin. The diversity in the structural forms shows a close relationship for its biological and pharmacological actions. Moreover, saponins showed antioxidant properties and the mechanism behind antimicrobial potential also elaborated in this review article is mainly because of the side sugar groups on these compounds. The collected data presented herein include chemical structures and molecular masses of all saponins so far. Their biological activity and therapeutic potential are also discussed. This information can be the starting point for future research on this important genus.
\end{abstract}

\section{Introduction}

Plants are well known for huge source of diverse array of phytochemicals. Flowering plant family Fabaceae is the largest and well spread family throughout the world, and
Medicago is one of its important genera comprising about 83 well-spread species, of which one-third are annuals [1]. The plants of the genus Medicago span diverse environmental conditions and are rich in alkaloids, flavonoids, naphthoquinones, and saponins [2-4]. Saponins comprise 
a huge range of glycosidic secondary metabolites reported in the genus Medicago [5]. Saponins are actually nonprotein amino acids. Complexes of polysaccharides and proteins such as lectins and enzyme inhibitors are also included in the saponins that behave as plant protectors. The genus Medicago is reported to possess higher levels of saponins, and these are named due to their foam-forming properties. In plants, saponins mainly occur in Medicago species classified into three classes, namely, steroid alkaloid glycosides, triterpene glycosides, and steroid glycosides [6].

Different Medicago species are reported to contain different kinds and variable amounts of saponins. Structurally diverse class of saponins may contain mono-, bi-, or tridesmosidic, linear or either branched by linking with the aglycone moiety (sapogenin) through an ether of ester bond. This structural diversity in saponins shows a huge range of hydrophobicity, acidity, and polarity which defines their multiple pharmacological as well as biological actions [6]. Many Medicago plant species are toxic for herbivores due to their higher saponins quantity, but Medicago saponins are reported to be toxic for birds and animals [7, 8].

Different parts, namely, roots, stem, leaves, and seeds, have different concentrations of various saponins. Keilbasa and colleagues extracted saponins from $M$. sativa leaves, roots, and sprouts and reported highest concentration of sapogenins in the roots, then sprouts and the least amounts in the leaves [9]. Moreover, it is further stressed that the extraction solvent and analytical technique may define the exact amounts of particular saponins in specific part of the Medicago plants. The edaphic factors of the Medicago plants also impart the production of specific saponins at all levels including the quantity [10]. Moreover, if the roots of M. truncatula species develop symbiotic relationship with certain mycorrhizal fungi, the concentration and amount of secondary metabolites including triterpene saponins also increase in $M$. truncatula plants having mycorrhizal association than those plants without mycorrhizal association [11]. In certain molecular engineering studies, the modulation of $\beta$-amyrin synthase-encoding sequence (AsOXA1) in $M$. truncatula showed raised biosynthesis of triterpene saponins and nodulation in roots [12]. Two M. truncatula cytochromes P450 enzymes (MtCYP72A67 and MtCYP72A68) along with $\beta$-amyrin synthase, MtCYP716A12 are involved in biosynthesis of saponins, and it was found that more saponins were produced due to the expression of these genes [13].

Among various natural phytochemicals, Medicago saponins are of great interest due to their wide spectrum pharmacological and biological properties [2]. These saponins in Medicago species are being formed when large molecules of triterpene glycosides get complexed with zahnic acid, hederagenin, medicagenic acid, bayogenin, and soyasapogenols $\mathrm{A}$ and $\mathrm{B}$ as the leading aglycones [14, 15]. These Medicago saponins are highly effective against tumorigenesis, fungal growth, and have cytotoxic effects for mollusks, bacteria, and various viruses $[6,16,17]$.
Keeping in view the diverse biological and pharmacological activities of saponins along with structural diversity, the most recent available literature about the saponins has been reviewed. Moreover, the nematicidal potential of saponins found in various Medicago species along with antioxidant properties shall also be discussed using latest literature to give an update of this important class of compounds. The collected data presented herein includes chemical structures and molecular masses of all saponins so far. Their biological activity and therapeutic potential are also discussed. This information can be the starting point for future research on this important genus.

\section{Extraction, Separation, Identification, and Quantification of Saponins}

Saponins are secondary plant metabolites distributed in the plant kingdom in several species, and they encompass triterpenoids, steroids, and steroidal alkaloids glycosylated having single or multiple sugar residues or chains [18]. Contents and composition profile of saponins depend on the cultivar, environmental conditions, physiological stage of growth, and plant organ. The saponin amount varied according to the species, ranging from $0.38 \pm 0.04 \%$ for M. rugosa Desrouss. to $1.35 \pm 0.08 \%$ for $M$. scutellate (L.) Mill. Medicagenic acid was the dominant aglycone in M. blancheana, M. doliata, M. littoralis, M. rotata, M. rugosa, M. scutellata, M. tornata, and M. truncatula, echinocystic acid in $M$. polymorpha, hederagenin and bayogenin in $M$. rigidula and $M$. arabica, and soyasapogenol B in M. aculeata [19]. The pharmaceutical property discoveries from the Medicago species have driven the emergence of various extraction technologies with the main purpose of maximizing the yield in order to accommodate the recent need. Therefore, Cheok et al. reviewed the extraction and quantification of saponins [20]. In general, the extraction techniques employed in saponin extraction are Soxhlet, maceration, and reflux extraction, microwave-assisted, ultrasound-assisted, and accelerated solvent extraction. The quantification of plant saponins is usually carried out by UV-spectrophotometric and chromatographic (HPLC, UPLC, TLC) methods [19]. Saponins are separated and purified from plant materials using chromatographic methods in many studies to identify a specific saponins compound and investigate its pharmaceutical property [20]. Sapogenins are usually obtained after acid hydrolysis of saponins and evaluated by GC/FID and GC/MS methods [19]. The elucidation and characterization of saponins structure are conducted usually on the basis of EI-MS, 1D, and 2D NMR data [20].

\section{Chemical Constituents}

For the genus Medicago, saponins make highly complex blend of glycosidic triterpenes originally derived from the isoprenoid pathway via the cyclization of 2,3-oxidosqualene to form $\beta$-amyrin nucleus. Oxidative modifications are driven by a series of cytochromes P450 (CYPs) and generate the aglycone moieties (sapogenins) that are subjected to 
glycosyl transfer reactions mediated by glycosyltransferases to give the different saponins. On this basis, 2 groups of saponins are reported in the $M$. sativa that can be differentiated: (1) sapogenins with $\mathrm{COOH}$ at the $\mathrm{C}-28$ and different oxidation states (zero, $\mathrm{OH}, \mathrm{CHO}, \mathrm{COOH}$ ) at $\mathrm{C}-23$ (medicagenic acid, zanhic acid, hederagenin, bayogenin, $2 \beta$, $3 \beta$-dihydroxy-23-oxo-olean-12-en-28-oic acid); and (2) sapogenins with an $\mathrm{OH}$ group at $\mathrm{C}-24$ with no substituent at C-28 (soyasapogenol A, B, E) [21]. Recently the queretaroic acid and its $2 \beta$-hydroxy derivative, $2 \beta, 3 \beta, 30$-trihydroxyolean-12-en-28-oic acid have been identified as novel aglycon in M. arabica (Figure 1). Queretaroic acid has the olean-12-ene skeleton and, together with glycyrrhetic acid, is one of the few naturally occurring triterpenes which is oxygenated at C-30 [22]. Queretaroic acid is supposed to be synthesized in vivo by a CYP P450 dependent hydroxylation of oleanolic acid [23].

In-depth examinations are conducted to elucidate chemical structures of saponins (compounds 1-95) in M. arabica, M. marina, $M$. polymorpha, $M$. truncatula, $M$. sativa, and $M$. arborea. Various saponins characterized till now from these species of Medicago are described in Tables 1-6. Various aboveground parts of M. arabica are well characterized to report the occurrence of saponins comprising of short chain sugar residues such as mono and bidesmosides of hederagenin, bayogenin, $2 \beta-$ hydroxy oleanolic acid, soyasapogenol B, and oleanolic acid. An exciting quality of saponins derived from $M$. arabica is bidesmosides of 3 $\beta$, 30-dihydroxyolean-12-en-28-oic acid and $2 \beta$, 3 $\beta$, 30-trihydroxyolean-12-en-28-oic acid (compounds $1-5$ ), as new aglycons for saponins of Medicago species (Table 1). All the detected saponins in M. marina are bidesmosidic compounds with the $\mathrm{C}-3$ position characterized by the presence of the same sugar, glucose or by the disaccharide chain Glc $(1 \longrightarrow 2)$ Glc (Table 2). Compounds 20, 21, 25, and 26 are undescribed in Medicago and never reported before in other plant species. Twelve triterpene saponins are recognized as glycosides of echinocystic acid hederagenin, soyasapogenol B, bayogenin, and caulophyllogenin in M. polymorpha (Table 3). Compounds 31 and 32 are declared as the novel natural compounds in Medicago species. Echinocystic acid is pioneer compounds to be reported in the genus Medicago. Saponins in $M$. truncatula seeds consist mainly of mono- and bidesmosides of soyasapogenol B and medicagenic acid (Table 4). Thirty-five pentacyclic triterpenoid saponins in $M$. sativa have been reported to occur as a complex mixture of short and long sugar chains of mono and bidesmosidic compounds having zanhic acid, bayogenin, hederagenin, medicagenic acid, $2 \beta, 3 \beta-$ Dihydroxy-23-oxo-olean-12en-28oic acid and soyasapogenol B (Table 5). Compounds 62, 77, 78, 84-88, and 91 are new triterpenoid saponins, but methyl ester derivative of saponins (compounds 77,78 , and 88 ) are accepted as artifacts examined through methanolic extraction [24]. $M$. arborea saponins from aerial parts are mainly mono and bidesmosides of medicagenic acid (Table 6).

\section{Biological Activity}

Being the model plant species, Medicago holds a prominent place in Leguminosae family mainly due to its saponins [36].
The presence of diverse class of chemicals holding multiple biological activities is all well reported and utilized for centuries. These saponins are primarily the glycosides having aglycone moiety which is formed involving enzymatic cyclization of 2,3 -oxidosqualene catalyzed by the $\beta$-amyrin cyclase [22]. Most of the Medicago species are being utilized as fodder for the grazing animals, but traditional medication system also clarifies that some of the species such as $M$. sativa herb are also beneficial for the human body. $M$. sativa is well recognized for centuries in traditional medication system in curing loss of memory, kidney issues, asthma, coughing, joint pains, and central nervous system disorders. All these pharmacological activities are detailed in the following text.

4.1. Insecticidal Activity. Due to increasing environmental and public health issues of using synthetic pesticides, the scientists are ever trying their hard to explore safer biological molecules to cure agricultural crops against multiple pathogens, namely, insects, bacterial, and fungal strains. Plant parasitic nematodes are cosmopolitan in distribution and are a major cause of huge economic losses for most of the agricultural crops and often quite hard to control the pathogens [37]. M. sativa L. shoot contains large amounts of saponins, which were identified in a recent study for their biological against aphid feeding, and found strong aphid inhibitory effects [38]. In an in vitro study, saponin rich mixtures of $M$. sativa showed effective growth inhibition on the viral vector nematodes like Xiphinema, the root-knot nematode Meloidogyne incognita, and Globodera rostochiensis which are the potato cyst parasites [37]. Three saponins, namely, 3-O-[ $\beta$-D-glucuronopyranosyl]-28$\mathrm{O}$-[ $\alpha$-L-rhamnopyranosyl $(1 \longrightarrow 2)$ - $\alpha$-L-arabinopyranosyl] medicagenic acid, Zanhic acid tridesmoside and 3-O$[\beta$-D-glucuronopyranosyl]-28-O- $\quad[\beta$-D-xylopyranosyl $(1 \longrightarrow 4)-\alpha$-L-rhamnopyranosyl $(1 \longrightarrow 2)-\alpha$-L-arabinopyranosyl] medicagenic acid were extracted from $M$. sativa L., potentially inhibits feeding of aphid Acyrthosiphon pisum assessed through electrical penetration graph technique in a dose dependent way [38]. In another recent study, saponins (10, $100 \mathrm{ppm}$ ) extracted from $M$. sativa extracts were applied freshly ecdysed $3^{\text {rd }}$ larval instar of Spodoptera littoralis, and higher dose $(100 \mathrm{ppm})$ caused absolute death while lower dose (10 ppm) caused only $26.7 \%$ mortalities [30]. Saponins mainly exert their effects by decreasing viability and rising mortalities, lowering the weights, reducing development and reproductive activities. Moreover, $M$. sativa saponins damaged the hindgut and fat body of $S$. littoralis badly to reduce its populations [30]. Another study examined the nematicidal effects of saponins of three different Medicago species ( $M$. sativa, $M$. arabica, $M$. arborea) using plant shoots and roots against Xiphinema index, which is a plant parasitic nematode. It is said that the presence of prosapogenins and sapogenins in shoots and roots extracts $(500 \mu \mathrm{g} / \mathrm{ml})$ effectively induces absolute $(100 \%)$ mortality of $X$. index, except the M. arborea that is less effective within 48 hours [39]. This nematicidal activity is correlated with the presence of aglycones (medicagenic acid and 


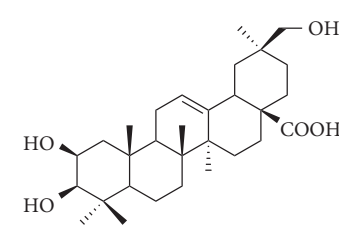

$2 \beta, 3 \beta, 30$-trihydroxyolean -12-en-28-oic acid

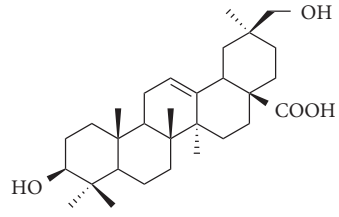

$3 \beta, 30$-dihydroxyolean -12-en-28-oic acid

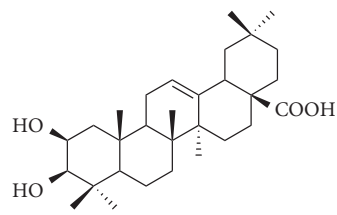

$2 \beta$-hydroxyoleanolic acid

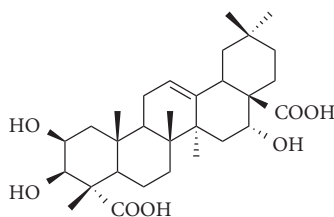

Zanhic acid

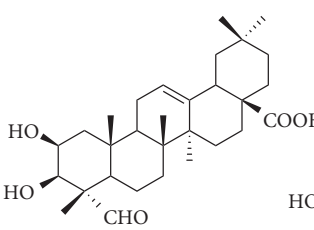

$2 \beta, 3 \beta$-dihydroxy-23-oxo -olean-12en-28oic acid<smiles>CC1(C)CCC2(C(=O)O)CC[C@]3(C)C(=CCC4[C@@]5(C)C[C@H](O)[C@@H](O)[C@](C)(C(=O)O)C5CC[C@]43C)C2C1</smiles>

Medicagenic acid

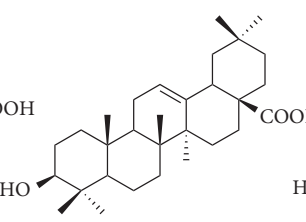

Oleanolic acid

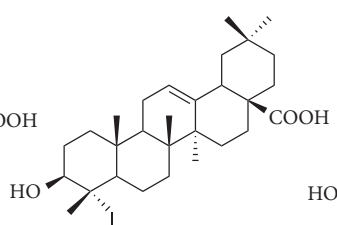

$\mathrm{OH}$

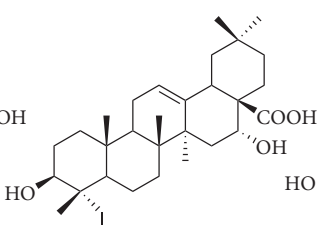

$\mathrm{OH}$

Hederagenin

Caulophyllogenin

Echinocystic acid

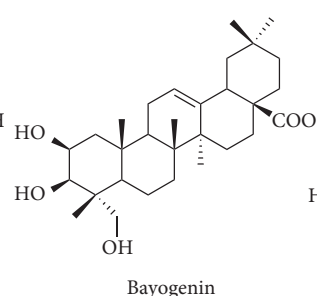

Bayogenin

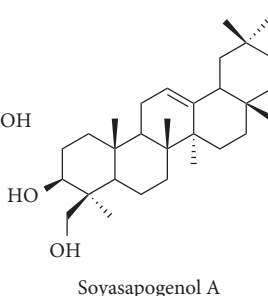

Soyasapogenol A
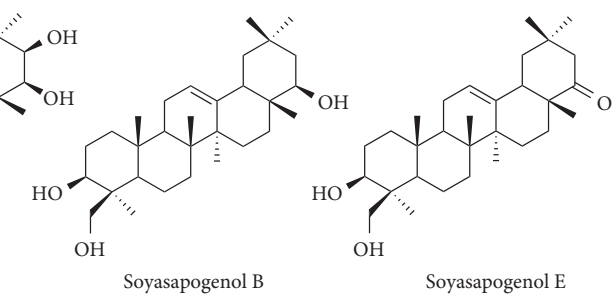

Figure 1: Chemical structure of sapogenins detected in Medicago species.

hederagenin) that occur in the roots and shoots saponin extracts [40]. M. truncatula saponins mediate caterpillar deterrence as a resistance mechanism in F83005.5 ecotype and associate these saponins as potential antifeedants that could be used in agricultural sustainable pest management strategies.

The seeds flour of $M$. truncatula showed a strong inhibition of the major pest (rice weevil Sitophilus oryzae) of cereals including rice [28], which were mainly responsible to the constituent of saponins 3-GlcA-28-AraRhaxylmedicagenate. Furthermore, when the saponin 3-GlcA-28-AraRhaxylmedicagenate was used in less concentration, it showed no effects on Caenorhabditis elegans (C. elegans) and E. coli, but at higher concentrations $(100 \mu \mathrm{g} / \mathrm{ml})$ it may lead to stopping the growth of Saccharomyces cerevisiae. Continuing this, the study emphasized the use of this target specific saponin (3-GlcA-28AraRhaxylmedicagenate) only for mature $S$. oryzae but not others like coleopteran Tribolium castaneum and the Sf9 insect cultured cells [28]. Root knot nematodes Meloidogyne incognita is the major cause of huge economic losses and is quiet hard to control. M. sativa L. crude extracts are much effective against tomato seedling infection caused by root knot nematode Meloidogyne incognita, which is mainly due to less cholesterol levels in root knot nematode eggs controlled by the saponins in plant extracts [40].

Gastrointestinal nematodes are considered as the crucial parasites in ruminants deteriorating the quality dairy products, hence appealing the exploration of natural phytochemicals bearing anthelmintic potential to avoid synthetic chemicals. The extracts of four Medicago species (M. sativa, M. arborea, M. polymorpha, M. polymorpha) were examined to find in vitro anthelminthic potential of
$1 \%$ saponins that cause a significant reduction $(>80 \%)$ in nematode egg hatching of gastrointestinal nematodes of dairy donkeys [41]. In another study, the Medicago plant extracts enriched with prosapogenins and saponins were tested for in vitro anthelmintic activity for sheep gastrointestinal strongyles (GISs) by the egg hatch test. The prosapogenins and saponins obtained from extracts of M. polymorpha cultivars Anglona showed strong inhibition on GIS eggs following a concentration-dependent manner [42].

4.2. Cytotoxic Effects. The saponins in alfalfa roots extracts $\left(50 \mu \mathrm{g} \mathrm{ml}^{-1}\right)$ induce over $75 \%$ cell death in poplar cells following a dose dependent fashion. This reduction in cell viability was mainly due to saponins-mediated induction of nitric oxide (NO) and reactive oxygen species (ROS) production, where the former found quite responsive to sodium azide and $\mathrm{N}^{G}$ monomethyl-L-arginine, which are the specific inhibitors of specific cellular pathways involved in NO biosynthesis in the plant cells isolated from poplar [43]. In another study, brine shrimps (Artemia Salina) were treated with extracts of twelve different Medicago plant species rich in a range of saponins. But, plant extracts of $M$. rigidula and $M$. arabica showed lethal dose ${ }_{50}$ of 4.6 and $10.1 \mu \mathrm{g} / \mathrm{mL}$, which depicts structure-activity relationship [19].

The different saponin extracts from $M$. arabica tops and roots showed best cytotoxic activity at the highest concentrations $(200 \mu \mathrm{g} / \mathrm{ml})$ against MCF-7 and HeLa cells using cisplatin as a positive control, and showed only 14 and $23 \%$ of cell survival, respectively. In this study, saponins 
Table 1: Saponins identified in $M$. arabica leaves.

\begin{tabular}{|c|c|c|c|c|c|c|}
\hline Aglycone & No. & 3-OH substituted & $\begin{array}{l}28-\mathrm{COOH} \\
\text { substituted }\end{array}$ & $30-\mathrm{CH}_{3}$ substituted & $\begin{array}{c}\text { Formula } \\
\text { weight }\end{array}$ & Ref. \\
\hline \multirow{3}{*}{$\begin{array}{l}2 \beta, 3 \beta, 30-\text { Trihydroxyolean-12- } \\
\text { en-28-oic acid }\end{array}$} & $1^{*}$ & $\alpha$-L-Ara $(1 \longrightarrow 2)-\beta-D-G l u A$ & - & $\beta$-D-Glc & $\begin{array}{l}\mathrm{C}_{47} \mathrm{H}_{74} \mathrm{O}_{20} \\
\quad 958\end{array}$ & {$[22]$} \\
\hline & $2^{*}$ & $\beta$-D-GluA & - & $\begin{array}{c}\alpha \text {-L-Ara }(1 \longrightarrow 2)- \\
\beta-\mathrm{D}-\mathrm{Glc}\end{array}$ & $\begin{array}{l}\mathrm{C}_{47} \mathrm{H}_{74} \mathrm{O}_{20} \\
\quad 958\end{array}$ & {$[22]$} \\
\hline & $3^{*}$ & $\beta$-D-GluA & - & $\beta$-D-Glc & $\begin{array}{c}\mathrm{C}_{42} \mathrm{H}_{66} \mathrm{O}_{16} \\
826 \\
\end{array}$ & {$[22]$} \\
\hline \multirow{2}{*}{$\begin{array}{l}3 \beta, 30 \text {-Dihydroxyolean-12-en- } \\
28 \text {-oic acid }\end{array}$} & $4^{*}$ & $\alpha$-L-Ara $(1 \longrightarrow 2)-\beta$-D-GluA & - & $\beta$-D-Glc & $\begin{array}{l}\mathrm{C}_{47} \mathrm{H}_{74} \mathrm{O}_{19} \\
942\end{array}$ & {$[22]$} \\
\hline & $5^{*}$ & $\beta$-D-GluA & - & $\left.\underset{\beta-\mathrm{L}-\mathrm{D}-\mathrm{Glc}}{\longrightarrow}{ }^{\alpha}\right)-$ & $\begin{array}{c}\mathrm{C}_{47} \mathrm{H}_{74} \mathrm{O}_{19} \\
942\end{array}$ & {$[22]$} \\
\hline \multirow{7}{*}{ Hederagenin } & 6 & $\begin{array}{l}\alpha \text {-L-Ara }(1 \longrightarrow 2)-\beta \text {-D-Glc- } \\
\quad(1 \longrightarrow 2)-\alpha \text {-L-Ara }\end{array}$ & $\beta$-D-Glc & - & $\begin{array}{c}\mathrm{C}_{52} \mathrm{H}_{84} \mathrm{O}_{22} \\
1060\end{array}$ & {$[22]$} \\
\hline & 7 & $\beta$-D-Glc- $(1 \longrightarrow 2)-\alpha$-L-Ara & $\beta$-D-Glc & - & $\begin{array}{c}\mathrm{C}_{47} \mathrm{H}_{76} \mathrm{O}_{18} \\
928\end{array}$ & {$[22]$} \\
\hline & 8 & $\alpha$-L-Ara & $\beta$-D-Glc & - & $\begin{array}{c}\mathrm{C}_{41} \mathrm{H}_{66} \mathrm{O}_{13} \\
766\end{array}$ & {$[22,25]$} \\
\hline & 9 & $\begin{array}{l}\alpha \text {-L-Ara }(1 \longrightarrow 2)-\beta \text {-D-Glc- } \\
\quad(1 \longrightarrow 2)-\alpha \text {-L-Ara }\end{array}$ & - & - & $\begin{array}{c}\mathrm{C}_{46} \mathrm{H}_{74} \mathrm{O}_{17} \\
898\end{array}$ & {$[22]$} \\
\hline & 10 & $\beta$-D-Glc- $(1 \longrightarrow 2)-\alpha$-L-Ara & - & - & $\begin{array}{l}\mathrm{C}_{41} \mathrm{H}_{66} \mathrm{O}_{13} \\
766\end{array}$ & {$[6,22,25]$} \\
\hline & 11 & $\alpha$-L-Ara $(1 \longrightarrow 2)-\beta$-D-GluA & - & - & $\begin{array}{c}\mathrm{C}_{41} \mathrm{H}_{64} \mathrm{O}_{14} \\
780\end{array}$ & {$[22]$} \\
\hline & 12 & $\alpha$-L-Ara & - & - & $\mathrm{C}_{35} \mathrm{H}_{56} \mathrm{O}_{8} 604$ & {$[22,25]$} \\
\hline \multirow[t]{2}{*}{ Bayogenin } & 13 & $\alpha$-L-Ara & $\beta$-D-Glc & - & $\begin{array}{c}\mathrm{C}_{41} \mathrm{H}_{64} \mathrm{O}_{14} \\
782\end{array}$ & {$[22,25]$} \\
\hline & 14 & $\alpha$-L-Ara & - & - & $\mathrm{C}_{35} \mathrm{H}_{56} \mathrm{O}_{9} 620$ & {$[22,25]$} \\
\hline \multirow{2}{*}{$2 \beta-H y d r o x y$ oleanolic acid } & 15 & $\alpha$-L-Ara $(1 \longrightarrow 2)-\beta$-D-GluA & $\beta$-D-Glc & - & $\begin{array}{l}\mathrm{C}_{47} \mathrm{H}_{74} \mathrm{O}_{19} \\
\quad 942\end{array}$ & {$[22]$} \\
\hline & 16 & $\beta$-D-GluA & - & - & $\begin{array}{c}\mathrm{C}_{36} \mathrm{H}_{56} \mathrm{O}_{10} \\
648 \\
\end{array}$ & {$[22]$} \\
\hline Soyasapogenol B & 17 & $\begin{array}{l}\text { L-Rha }(1 \longrightarrow 2)-\beta \text {-D-Gal- } \\
\quad(1 \longrightarrow 2)-\beta \text {-D-GluA }\end{array}$ & - & - & $\begin{array}{c}\mathrm{C}_{48} \mathrm{H}_{78} \mathrm{O}_{18} \\
942 \\
\end{array}$ & {$[6,22]$} \\
\hline \multirow[t]{2}{*}{ Oleanolic acid } & 18 & $\alpha$-L-Ara $(1 \longrightarrow 2)-\beta$-D-GluA & - & - & $\begin{array}{c}\mathrm{C}_{41} \mathrm{H}_{64} \mathrm{O}_{13} \\
764\end{array}$ & {$[22]$} \\
\hline & 19 & $\beta$-D-GluA & - & - & $\mathrm{C}_{36} \mathrm{H}_{56} \mathrm{O}_{9} 632$ & {$[22]$} \\
\hline
\end{tabular}

TABLE 2: Saponins from M. marina leaves and roots.

\begin{tabular}{|c|c|c|c|c|c|}
\hline Aglycone & No. & 3-OH substituted & 28-COOH substituted & Formula weight & Ref. \\
\hline \multirow{5}{*}{ Zanhic acid } & $20^{*}$ & $\beta-\mathrm{D}-\mathrm{Glc}(1 \longrightarrow 2)-\beta-\mathrm{D}-\mathrm{Glc}$ & $\begin{array}{c}\beta-\mathrm{D}-\mathrm{Xyl}(1 \longrightarrow 4)-[\beta-\mathrm{D}-\mathrm{Api}(1 \longrightarrow 3)]-\alpha-\mathrm{L}- \\
\mathrm{Rha}(1 \stackrel{\longrightarrow}{\longrightarrow})-\alpha-\mathrm{L}-\mathrm{Ara}\end{array}$ & $\begin{array}{c}\mathrm{C}_{63} \mathrm{H}_{100} \mathrm{O}_{33} \\
1384\end{array}$ & {$[26]$} \\
\hline & $21^{*}$ & $\beta-\mathrm{D}-\mathrm{Glc}(1 \longrightarrow 2)-\beta-\mathrm{D}-\mathrm{Glc}$ & $\beta$-D-Xyl $(1 \longrightarrow 4)-\alpha-\mathrm{L}-\mathrm{Rha}(1 \longrightarrow 2)-\alpha$-L-Ara & $\mathrm{C}_{58} \mathrm{H}_{92} \mathrm{O}_{29} 1252$ & [26] \\
\hline & 22 & $\beta$-D-Glc $(1 \longrightarrow 2)-\beta$-D-Glc & $\begin{array}{c}\beta-\mathrm{D}-\mathrm{Xyl}(1 \longrightarrow 4)-[\beta-\mathrm{D}-\operatorname{Ara}(1 \longrightarrow 3)]-\alpha-\mathrm{L}- \\
\operatorname{Rha}(1 \stackrel{\longrightarrow}{\longrightarrow})-\alpha-\mathrm{L}-\operatorname{Ara}\end{array}$ & $\begin{array}{c}\mathrm{C}_{63} \mathrm{H}_{100} \mathrm{O}_{33} \\
1384\end{array}$ & [26] \\
\hline & 23 & $\beta$-D-Glc & $\begin{array}{c}\beta-\mathrm{D}-\mathrm{Xyl}(1 \longrightarrow 4)-[\beta-\mathrm{D}-\mathrm{Ara}(1 \longrightarrow 3)]-\alpha-\mathrm{L}- \\
\operatorname{Rha}(1 \longrightarrow 2)-\alpha-\mathrm{L}-\mathrm{Ara}\end{array}$ & $\mathrm{C}_{57} \mathrm{H}_{90} \mathrm{O}_{28} 1222$ & [26] \\
\hline & 24 & $\beta$-D-Glc & $\beta$-D-Xyl $(1 \longrightarrow 4)-\alpha$-L-Rha $(1 \longrightarrow 2)-\alpha$-L-Ara & $\mathrm{C}_{52} \mathrm{H}_{82} \mathrm{O}_{24} 1090$ & [26] \\
\hline \multirow{4}{*}{$\begin{array}{l}\text { Medicagenic } \\
\text { acid }\end{array}$} & $25^{*}$ & $\beta$-D-Glc $(1 \longrightarrow 2)-\beta$-D-Glc & $\begin{array}{c}\beta-\mathrm{D}-\mathrm{Xyl}(1 \longrightarrow 4)-[\beta-\mathrm{D}-\operatorname{Ara}(1 \longrightarrow 3)]-\alpha-\mathrm{L}- \\
\mathrm{Rha}(1 \longrightarrow 2)-\alpha-\mathrm{L}-\mathrm{Ara}\end{array}$ & $\begin{array}{c}\mathrm{C}_{63} \mathrm{H}_{100} \mathrm{O}_{32} \\
1368\end{array}$ & [26] \\
\hline & $26^{*}$ & $\beta$-D-Glc & $\begin{array}{c}\beta-\mathrm{D}-\mathrm{Xyl}(1 \longrightarrow 4)-[\beta-\mathrm{D}-\operatorname{Ara}(1 \longrightarrow 3)]-\alpha-\mathrm{L}- \\
\operatorname{Rha}(1 \longrightarrow 2)-\alpha-\mathrm{L}-\mathrm{Ara}\end{array}$ & $\mathrm{C}_{57} \mathrm{H}_{90} \mathrm{O}_{27} 1206$ & [26] \\
\hline & 27 & $\beta$-D-Glc $(1 \longrightarrow 2)-\beta-\mathrm{D}-\mathrm{Glc}$ & $\begin{array}{c}\beta-\mathrm{D}-\mathrm{Xyl}(1 \longrightarrow 4)-[\beta-\mathrm{D}-\mathrm{Api}(1 \longrightarrow 3)]-\alpha-\mathrm{L}- \\
\operatorname{Rha}(1 \longrightarrow 2)-\alpha-\mathrm{L}-\mathrm{Ara}\end{array}$ & $\begin{array}{c}\mathrm{C}_{63} \mathrm{H}_{100} \mathrm{O}_{32} \\
1368\end{array}$ & [26] \\
\hline & 28 & $\beta$-D-Glc & $\beta$-D-Xyl $(1 \longrightarrow 4)-\alpha$-L-Rha $(1 \longrightarrow 2)-\alpha$-L-Ara & $\mathrm{C}_{52} \mathrm{H}_{82} \mathrm{O}_{23} 1074$ & [26] \\
\hline Soyasapogenol B & 29 & $\alpha$-L-Rha $(1 \longrightarrow \underset{\beta-D-G l u A}{\longrightarrow})-\beta$-Gal $(1 \longrightarrow 2)-$ & - & $\mathrm{C}_{48} \mathrm{H}_{78} \mathrm{O}_{18} 942$ & {$[26]$} \\
\hline Soyasapogenol E & 30 & $\alpha$-L-Rha $(1 \underset{\beta-D-G l u A}{\longrightarrow})-\beta$-D-Gal $(1 \longrightarrow 2)-$ & - & $\mathrm{C}_{48} \mathrm{H}_{76} \mathrm{O}_{18} 940$ & [26] \\
\hline
\end{tabular}


TABle 3: Saponins from M. polymorpha leaves and roots.

\begin{tabular}{|c|c|c|c|c|c|}
\hline Aglycone & No. & 3-OH substituted & 28-COOH substituted & Formula weight & Ref. \\
\hline \multirow{5}{*}{ Echinocystic acid } & $31^{*}$ & $\alpha$-L-Ara & $\beta$-D-Glc & $\mathrm{C}_{41} \mathrm{H}_{66} \mathrm{O}_{13} 766$ & [27] \\
\hline & $32^{*}$ & $\alpha$-L-Ara & $\beta$-D-Glc $(1 \longrightarrow 6)-\beta$-D-Glc & $\mathrm{C}_{47} \mathrm{H}_{76} \mathrm{O}_{18} 928$ & [27] \\
\hline & 33 & $\beta$-D-Glc $(1 \longrightarrow 2)-\alpha$-L-Ara & $\beta-\mathrm{D}-\mathrm{Glc}(1 \longrightarrow 6)-\beta-\mathrm{D}-\mathrm{Glc}$ & $\mathrm{C}_{53} \mathrm{H}_{86} \mathrm{O}_{23} 1090$ & [27] \\
\hline & 34 & $\alpha-\mathrm{L}-\mathrm{Ara}$ & - & $\mathrm{C}_{35} \mathrm{H}_{56} \mathrm{O}_{8} 604$ & [27] \\
\hline & 35 & $\beta$-D-Glc & - & $\mathrm{C}_{36} \mathrm{H}_{58} \mathrm{O}_{9} 634$ & {$[25]$} \\
\hline \multirow{5}{*}{ Hederagenin } & 36 & $\alpha$-L-Rha(1 $\longrightarrow 2)-\alpha$-L-Ara & $\beta$-D-Glc $(1 \longrightarrow 6)-\beta$-D-Glc & $\mathrm{C}_{53} \mathrm{H}_{86} \mathrm{O}_{22} 1074$ & {$[27]$} \\
\hline & 37 & $\beta$-D-Glc $(1 \longrightarrow 2)-\alpha$-L-Ara & $\beta-\mathrm{D}-\mathrm{Glc}$ & $\mathrm{C}_{47} \mathrm{H}_{76} \mathrm{O}_{18} 928$ & [27] \\
\hline & 38 & $\alpha-\mathrm{L}-\mathrm{Ara}$ & $\beta$-D-Glc & $\mathrm{C}_{41} \mathrm{H}_{66} \mathrm{O}_{13} 766$ & [27] \\
\hline & 39 & $\alpha$-L-Rha $(1 \longrightarrow 2)-\alpha$-L-Ara & - & $\mathrm{C}_{41} \mathrm{H}_{66} \mathrm{O}_{12} 750$ & {$[25,27]$} \\
\hline & 40 & $\alpha$-L-Ara & - & $\mathrm{C}_{35} \mathrm{H}_{56} \mathrm{O}_{8} 604$ & {$[27]$} \\
\hline Soyasapogenol B & 41 & $\alpha$-L-Rha $(1 \longrightarrow 2)-\beta$-D-Gal $(1 \longrightarrow 2)-\beta$-D-GluA & - & $\mathrm{C}_{48} \mathrm{H}_{78} \mathrm{O}_{18} 942$ & [27] \\
\hline Caulophyllogenin & 42 & $\alpha$-L-Ara & - & $\mathrm{C}_{35} \mathrm{H}_{56} \mathrm{O}_{9} 620$ & [27] \\
\hline Bayogenin & 43 & $\alpha$-L-Ara & - & $\mathrm{C}_{35} \mathrm{H}_{56} \mathrm{O}_{9} 620$ & [27] \\
\hline
\end{tabular}

TABle 4: Saponins from M. truncatula seeds.

\begin{tabular}{|c|c|c|c|c|c|}
\hline Aglycone & No. & 3-OH substituted & 28-COOH substituted & Formula weight & Ref. \\
\hline Soyasapogenol B & $\begin{array}{l}44 \\
45\end{array}$ & $\begin{array}{c}\beta-\mathrm{D}-\mathrm{GlcA} \\
\mathrm{L}-\mathrm{Rha}(1 \longrightarrow 2)-\beta-\mathrm{D}-\mathrm{Gal}(1 \longrightarrow 2)-\beta-\mathrm{D}-\mathrm{GlcA}\end{array}$ & $\begin{array}{c}3-\mathrm{D}-\mathrm{Xyl}(1 \longrightarrow 4)-\alpha \text {-L-Rha }(1 \longrightarrow 2)-\alpha \text {-L-Ara } \\
-\end{array}$ & & $\begin{array}{l}{[28]} \\
{[29]}\end{array}$ \\
\hline Medicagenic acid & $\begin{array}{l}46 \\
47 \\
48\end{array}$ & $\begin{array}{c}\beta \text {-D-GlcA } \\
\beta \text {-D-Glc } \\
\beta \text {-D-Glc }\end{array}$ & $\begin{array}{c}\alpha \text {-L-Ara }(1 \longrightarrow 2)-\alpha \text {-L-Rha }(1 \longrightarrow 2)-\beta \text {-D-Xyl } \\
\alpha \text {-L-Ara }(1 \longrightarrow 2)-\alpha \text {-L-Rha }(1 \longrightarrow 2)-\beta \text {-D-Xyl } \\
\beta \text {-D-Glc }\end{array}$ & $\begin{array}{l}\mathrm{C}_{52} \mathrm{H}_{80} \mathrm{O}_{24} 1088 \\
\mathrm{C}_{52} \mathrm{H}_{82} \mathrm{O}_{23} 1074 \\
\mathrm{C}_{42} \mathrm{H}_{66} \mathrm{O}_{16} 826\end{array}$ & $\begin{array}{l}{[29]} \\
{[29]} \\
{[29]}\end{array}$ \\
\hline
\end{tabular}

(monodesmosides of hederagenin and bayogenin) rich plant extracts mainly containing $1,3-O-\beta$-D-glucopyranosyl $(1 \longrightarrow 2)-\alpha$-L-arabinopyranosyl hederagenin potentially reduced the proliferation of MCF-7 and HeLa cells at 24 hours.

4.3. Antioxidant Potential. The extraction studies reported that Medicago plants extracts bear strong antioxidant potential. For instance, various parts (roots, stem, leaves) of $M$. sativa plant ethanolic extracts yield various phenolics, flavonoids, and saponins, all of which show higher antioxidant potential [44]. M. lupulina is comparatively less studied species, and its crude methanolic extracts showed antioxidant activity with a Trolox ${ }^{\circledR}$ equivalent antioxidant activity (TEAA) and ferric reducing antioxidant power (FRAP) values of $45.4 \mu \mathrm{mol}$ Trolox/g dw and $0.2 \mathrm{mmol} \mathrm{Fe}^{2+} /$ $\mathrm{g} \mathrm{dw}$ through DPPH and FRAP assay [45]. In vitro free radical scavenging activity using DPPH assay was performed using various extracts of $M$. sativa seeds, but ethanolic extracts of seeds and seed sprouts showed maximum and ascending radical scavenging activity in a concentration dependent fashion $(10,20,30,40,50,60,70,80,90,100 \mu \mathrm{g})$ [46].

4.4. Antimicrobial Effects. The extracts of $M$. sativa have strong inhibitory effect on Proteus vulgaris, Escherichia coli (E. coli), Klebsiella pneumonia, Salmonella typhi, Mucor circinelloides, Rhizopus azygosporus, and R. microsporus with less pronounced action on Shigella flexneri, Staphylococcus epidermidis, Candida albicans, and Emericella quadrillineata [10]. Moreover, a reversed influence on Pseudomonas aerugenosa and Streptococcus pyrogenes was seen, while
Pseudallescheria ellipsoidea, two species of Penicillium, and five of Aspergillus were seen somewhat resistant for these plant extracts [10].

$M$. sativa plant extracts rich in saponins showed strong antifungal potential to successfully check the growth of Candida albicans along with certain clinical pathogenic fungal strains mainly by inhibiting the germ tube formation, retarded the growth of fungal hyphae, and lessened the adherence of yeast cells and eradication of biofilm development at 24 hours after treatment [47]. It is further stated that saponin extracts of $M$. sativa in a dosage range harmful to check the growth of fungi are least toxic to the mice fibroblast L929 cells, which showed them being safe to use for human antifungal conditions [47].

4.5. Miscellaneous Effects. The excessive accumulation of ROS at cellular level along with chronic disregulation of cellular antioxidant defense systems leads to diverse pathologies of certain neurodegenerative issues such as Parkinson's disease, amyotrophic lateral sclerosis, Alzheimer's disease, and Huntington disease etc. Li and colleagues isolated three pentacyclic triterpenoid saponins along with medicagenic acid from $M$. sativa using 70\% ethanolic extraction and studied their neuroprotective effects using human neuroblastoma SHSY5Y cells [35].

Peroxisome proliferator-activated receptor $(\operatorname{PPAR} \gamma)$ is an important regulator of glucose and lipid homeostasis as well as an important pharmacological target for treating metabolic diseases. Saponins and sapogenins found in Medicago species showed antagonist potential against PPAR $\gamma$, which could be helpful to restrain differentiation in 
TABle 5: Saponins from M. sativa.

\begin{tabular}{|c|c|c|c|c|c|}
\hline Aglycone & No. & 3-OH substituted & 28-COOH substituted & $\begin{array}{c}\text { Formula } \\
\text { weight }\end{array}$ & Ref. \\
\hline \multirow{15}{*}{ Medicagenic acid } & 49 & $\beta-\mathrm{D}-\mathrm{GlcA}$ & $\alpha$-L-Rha $(1 \longrightarrow 2)-\alpha$-L-Ara & $\begin{array}{c}\mathrm{C}_{47} \mathrm{H}_{72} \mathrm{O}_{20} \\
956\end{array}$ & {$[30]$} \\
\hline & 50 & $\begin{array}{l}\alpha \text {-L-Rha }(1 \longrightarrow 2)-\beta \text {-D- } \\
\text { Gal }(1 \longrightarrow 2)-\beta \text {-D-GluA }\end{array}$ & - & $\begin{array}{c}\mathrm{C}_{48} \mathrm{H}_{74} \mathrm{O}_{21} \\
986\end{array}$ & {$[31]$} \\
\hline & 51 & $\beta-\mathrm{D}-\mathrm{GlcA}$ & $\begin{array}{c}\beta \text {-D-Xyl }(1 \longrightarrow 4)-\alpha \text {-L-Rha }(1 \longrightarrow 2)- \\
\alpha \text {-L-Ara }\end{array}$ & $\begin{array}{c}\mathrm{C}_{52} \mathrm{H}_{80} \mathrm{O}_{24} \\
1088\end{array}$ & {$[18,31]$} \\
\hline & 52 & $\beta$-D-GlcA & $\beta$-D-GlcA & $\begin{array}{c}\mathrm{C}_{42} \mathrm{H}_{62} \mathrm{O}_{18} \\
854\end{array}$ & {$[32]$} \\
\hline & 53 & $\beta-\mathrm{D}-\mathrm{Glc}(1 \longrightarrow 3)-\beta-\mathrm{D}-\mathrm{Glc}$ & $\alpha$-L-Rha $(1 \longrightarrow 2)-\alpha$-L-Ara & $\begin{array}{c}\mathrm{C}_{53} \mathrm{H}_{84} \mathrm{O}_{24} \\
1104\end{array}$ & {$[32]$} \\
\hline & 54 & $\beta$-D-GlcA & $\begin{array}{c}\beta \text {-D-Api }(1 \longrightarrow 3)-[\beta-\mathrm{D}- \\
\mathrm{Xyl}(1 \longrightarrow 4)]-\alpha \text {-L-Rha }(1 \longrightarrow 2)- \\
\alpha \text {-L-Ara }\end{array}$ & $\begin{array}{c}\mathrm{C}_{57} \mathrm{H}_{88} \mathrm{O}_{28} \\
1220\end{array}$ & {$[32]$} \\
\hline & 55 & $\beta$-D-Glc & $\begin{array}{c}\beta \text {-D-Glc }(1 \longrightarrow 4)-\alpha \text {-L-Rha }(1 \longrightarrow 2)- \\
\alpha \text {-L-Ara }\end{array}$ & $\begin{array}{c}\mathrm{C}_{53} \mathrm{H}_{84} \mathrm{O}_{24} \\
1104\end{array}$ & {$[33]$} \\
\hline & 56 & $\beta$-D-Glc $(1 \longrightarrow 2)-\beta-\mathrm{D}-\mathrm{Glc}$ & $\begin{array}{c}\beta \text {-D-Xyl }(1 \longrightarrow 4)-\alpha \text {-L-Rha }(1 \longrightarrow 2)- \\
\alpha \text {-L-Ara }\end{array}$ & $\begin{array}{c}\mathrm{C}_{58} \mathrm{H}_{92} \mathrm{O}_{28} \\
1236\end{array}$ & {$[18,33]$} \\
\hline & 57 & $\begin{array}{l}\alpha \text {-L-Ara }(1 \longrightarrow 2)-\beta-\mathrm{D}- \\
\operatorname{Glc}(1 \longrightarrow 2)-\alpha-\mathrm{L}-\mathrm{Ara}\end{array}$ & $\beta$-D-Glc & $\begin{array}{c}\mathrm{C}_{52} \mathrm{H}_{82} \mathrm{O}_{24} \\
1090\end{array}$ & {$[33]$} \\
\hline & 58 & $\beta$-D-Glc & - & $\begin{array}{c}\mathrm{C}_{36} \mathrm{H}_{56} \mathrm{O}_{11} \\
664\end{array}$ & {$[1,18,25]$} \\
\hline & 59 & $\beta$-D-Glc & $\beta$-D-Glc & $\begin{array}{c}\mathrm{C}_{42} \mathrm{H}_{66} \mathrm{O}_{16} \\
826\end{array}$ & {$[1,18,25,34]$} \\
\hline & 60 & $\beta$-D-Glc & $\begin{array}{c}\beta-\mathrm{D}-\mathrm{Xyl}(1 \longrightarrow 4)-\alpha-\mathrm{L}-\mathrm{Rha}(1 \longrightarrow 2)- \\
\alpha-\mathrm{L}-\mathrm{Ara}\end{array}$ & $\begin{array}{c}\mathrm{C}_{52} \mathrm{H}_{82} \mathrm{O}_{23} \\
1074\end{array}$ & {$[1,6,18]$} \\
\hline & 61 & $\beta$-D-Glc $(1 \longrightarrow 2)-\beta-\mathrm{D}-\mathrm{Glc}$ & $\beta$-D-Glc & $\begin{array}{c}\mathrm{C}_{48} \mathrm{H}_{76} \mathrm{O}_{21} \\
988\end{array}$ & {$[18,25]$} \\
\hline & $62^{*}$ & $\begin{array}{l}\beta \text {-D-Glc }(1 \longrightarrow 2)-\beta \text {-D- } \\
\text { Glc }(1 \longrightarrow 2)-\beta-D-G l c\end{array}$ & - & $\begin{array}{c}\mathrm{C}_{48} \mathrm{H}_{76} \mathrm{O}_{21} \\
988\end{array}$ & {$[34]$} \\
\hline & 63 & $\begin{array}{l}\alpha \text {-L-Rha }(1 \longrightarrow 2)-\beta \text {-D- } \\
\text { Glc }(1 \longrightarrow 2)-\beta \text {-D-Glc }\end{array}$ & - & $\begin{array}{c}\mathrm{C}_{48} \mathrm{H}_{76} \mathrm{O}_{20} \\
972 \\
\end{array}$ & {$[34]$} \\
\hline \multirow{12}{*}{ Zanhic acid } & 64 & $\beta$-D-GlcA & $\begin{array}{c}\beta \text {-D-Xyl }(1 \longrightarrow 4)-\alpha \text {-L-Rha }(1 \longrightarrow 2)- \\
\alpha \text {-L-Ara }\end{array}$ & $\begin{array}{c}\mathrm{C}_{52} \mathrm{H}_{80} \mathrm{O}_{25} \\
1104\end{array}$ & {$[30]$} \\
\hline & 65 & $\begin{array}{l}\beta-\mathrm{D}-\mathrm{Xyl}(1 \longrightarrow 4)-\alpha-\mathrm{L}- \\
\operatorname{Rha}(1 \longrightarrow 2)-\alpha-\mathrm{L}-\text { Ara }\end{array}$ & - & $\begin{array}{c}\mathrm{C}_{46} \mathrm{H}_{72} \mathrm{O}_{19} \\
928\end{array}$ & {$[32]$} \\
\hline & 66 & $\beta$-D-Glc & $\begin{array}{c}\beta \text {-D-Xyl }(1 \longrightarrow 4)-\alpha \text {-L-Rha }(1 \longrightarrow 2)- \\
\alpha \text {-L-Ara }\end{array}$ & $\begin{array}{c}\mathrm{C}_{52} \mathrm{H}_{82} \mathrm{O}_{24} \\
1090\end{array}$ & {$[32]$} \\
\hline & 67 & $\beta$-D-Glc $(1 \longrightarrow 3)-\beta-\mathrm{D}-\mathrm{Glc}$ & $\alpha$-L-Rha $(1 \longrightarrow 2)-\alpha$-L-Ara & $\begin{array}{c}\mathrm{C}_{53} \mathrm{H}_{84} \mathrm{O}_{25} \\
1120\end{array}$ & {$[32]$} \\
\hline & 68 & $\beta$-D-Glc & $\begin{array}{c}\alpha-\mathrm{L}-\operatorname{Ara}(1 \longrightarrow 3)-[\beta-\mathrm{D}- \\
\operatorname{Xyl}(1 \longrightarrow 4)]-\alpha-\mathrm{L}-\mathrm{Rha}(1 \longrightarrow 2)- \\
\alpha \text {-L-Ara }\end{array}$ & $\begin{array}{c}\mathrm{C}_{57} \mathrm{H}_{90} \mathrm{O}_{28} \\
1222\end{array}$ & {$[32]$} \\
\hline & 69 & $\beta$-D-GlcA & $\begin{array}{c}\alpha \text {-L-Ara }(1 \longrightarrow 3)-[\beta-\mathrm{D}- \\
\operatorname{Xyl}(1 \longrightarrow 4)]-\alpha \text {-L-Rha }(1 \longrightarrow 2)- \\
\alpha \text {-L-Ara }\end{array}$ & $\begin{array}{c}\mathrm{C}_{57} \mathrm{H}_{88} \mathrm{O}_{29} \\
1236\end{array}$ & {$[32]$} \\
\hline & 70 & $\beta$-D-Glc $(1 \longrightarrow 3)-\beta-\mathrm{D}-\mathrm{Glc}$ & $\begin{array}{c}\alpha \text {-L-Ara }(1 \longrightarrow 3)-\alpha \text {-L-Rha }(1 \longrightarrow 2)- \\
\alpha \text {-L-Ara }\end{array}$ & $\begin{array}{c}\mathrm{C}_{58} \mathrm{H}_{92} \mathrm{O}_{29} \\
1252\end{array}$ & {$[32]$} \\
\hline & 71 & $\beta$-D-Glc $(1 \longrightarrow 2)-\beta-\mathrm{D}-\mathrm{Glc}$ & $\begin{array}{c}\alpha \text {-L-Ara }(1 \longrightarrow 3)-[\beta-\mathrm{D}- \\
\operatorname{Xyl}(1 \longrightarrow 4)]-\alpha \text {-L-Rha }(1 \longrightarrow 2)- \\
\alpha \text {-L-Ara }\end{array}$ & $\begin{array}{c}\mathrm{C}_{63} \mathrm{H}_{100} \mathrm{O}_{33} \\
1384\end{array}$ & {$[32]$} \\
\hline & 72 & $\begin{array}{l}\alpha-\mathrm{L}-\operatorname{Ara}(1 \longrightarrow 2)-\beta-\mathrm{D}- \\
\operatorname{Glc}(1 \longrightarrow 2)-\beta-\mathrm{D}-\mathrm{Glc}\end{array}$ & $\begin{array}{c}\beta \text {-D-Api }(1 \longrightarrow 3)-[\beta-\mathrm{D}- \\
\operatorname{Xyl}(1 \longrightarrow 4)]-\alpha \text {-L-Rha }(1 \longrightarrow 2)- \\
\alpha \text {-L-Ara }\end{array}$ & $\begin{array}{c}\mathrm{C}_{68} \mathrm{H}_{108} \mathrm{O}_{37} \\
1516\end{array}$ & {$[32]$} \\
\hline & 73 & $\begin{array}{l}\beta-\mathrm{D}-\mathrm{Glc}(1 \longrightarrow 2)-\beta-\mathrm{D}- \\
\operatorname{Glc}(1 \longrightarrow 2)-\beta-\mathrm{D}-\mathrm{Glc}\end{array}$ & $\begin{array}{c}\beta \text {-D-Xyl }(1 \longrightarrow 4)-\alpha \text {-L-Rha }(1 \longrightarrow 2)- \\
\alpha \text {-L-Ara }\end{array}$ & $\begin{array}{c}\mathrm{C}_{64} \mathrm{H}_{102} \mathrm{O}_{34} \\
1414\end{array}$ & {$[1,18,25]$} \\
\hline & 74 & $\begin{array}{l}\beta \text {-D-Glc }(1 \longrightarrow 2)-\beta-D- \\
\text { Glc }(1 \longrightarrow 2)-\beta-D-G l c\end{array}$ & $\begin{array}{c}\beta \text {-D-Api }(1 \longrightarrow 3)-[\beta-\mathrm{D}- \\
\mathrm{Xyl}(1 \longrightarrow 4)]-\alpha \text {-L-Rha }(1 \longrightarrow 2)- \\
\alpha \text {-L-Ara }\end{array}$ & $\begin{array}{c}\mathrm{C}_{69} \mathrm{H}_{110} \mathrm{O}_{38} \\
1546\end{array}$ & {$[18,30]$} \\
\hline & 75 & $\beta$-D-GluA & $\begin{array}{c}\alpha \text {-L-Ara }(1 \longrightarrow 3)-[\beta-\mathrm{D}- \\
\operatorname{Xyl}(1 \longrightarrow 4)]-\alpha \text {-L-Rha }(1 \longrightarrow 2)- \\
\alpha \text {-L-Ara }\end{array}$ & $\begin{array}{c}\mathrm{C}_{57} \mathrm{H}_{88} \mathrm{O}_{29} \\
1236\end{array}$ & {$[1]$} \\
\hline
\end{tabular}


TABle 5: Continued.

\begin{tabular}{|c|c|c|c|c|c|}
\hline Aglycone & No. & 3-OH substituted & 28-COOH substituted & $\begin{array}{c}\text { Formula } \\
\text { weight }\end{array}$ & Ref. \\
\hline \multirow{3}{*}{ Bayogenin } & 76 & $\alpha$-L-Ara & $\beta$-D-Glc & $\begin{array}{c}\mathrm{C}_{41} \mathrm{H}_{66} \mathrm{O}_{14} \\
782\end{array}$ & {$[32]$} \\
\hline & $77^{*}$ & $\begin{array}{l}\beta-\mathrm{D}-\mathrm{Gal}(1 \longrightarrow 2)-\beta-\mathrm{D}- \\
\text { GluAME }\end{array}$ & $\beta$-D-Glu & $\begin{array}{c}\mathrm{C}_{49} \mathrm{H}_{76} \mathrm{O}_{22} \\
1016\end{array}$ & {$[35]$} \\
\hline & $78^{*}$ & $\begin{array}{l}\beta-\mathrm{D}-\mathrm{Xyl}(1 \longrightarrow 4)-\beta-\mathrm{D}- \\
\text { GluAME }\end{array}$ & $\beta$-D-Glc & $\begin{array}{l}\mathrm{C}_{48} \mathrm{H}_{76} \mathrm{O}_{20} \\
\quad 972\end{array}$ & {$[35]$} \\
\hline \multirow{11}{*}{ Hederagenin } & 79 & - & $\beta$-D-Glc & $\mathrm{C}_{36} \mathrm{H}_{58} \mathrm{O}_{9} 634$ & {$[32]$} \\
\hline & 80 & $\alpha$-L-Ara $(1 \longrightarrow 2)-\beta-\mathrm{D}-\mathrm{Glc}$ & $\beta$-D-Glc & $\begin{array}{c}\mathrm{C}_{47} \mathrm{H}_{76} \mathrm{O}_{18} \\
928\end{array}$ & {$[18]$} \\
\hline & 81 & $\begin{array}{l}\alpha \text {-L-Ara }(1 \longrightarrow 2)-\beta-\mathrm{D}- \\
\operatorname{Glc}(1 \longrightarrow 2)-\alpha \text {-L-Ara }\end{array}$ & - & $\begin{array}{c}\mathrm{C}_{46} \mathrm{H}_{74} \mathrm{O}_{17} \\
898\end{array}$ & {$[18]$} \\
\hline & 82 & $\beta$-D-Glc $(1 \longrightarrow 2)-\alpha$-L-Ara & - & $\begin{array}{l}\mathrm{C}_{41} \mathrm{H}_{66} \mathrm{O}_{13} \\
766\end{array}$ & [1] \\
\hline & 83 & $\begin{array}{l}\alpha \text {-L-Ara }(1 \longrightarrow 2)-\beta \text {-D- } \\
\text { Glc }(1 \longrightarrow 2)-\alpha \text {-L-Ara }\end{array}$ & $\beta$-D-Glc & $\begin{array}{c}\mathrm{C}_{52} \mathrm{H}_{84} \mathrm{O}_{22} \\
1060\end{array}$ & {$[18,34]$} \\
\hline & $84^{*}$ & $\beta$-D-Xyl $(1 \longrightarrow 3)-\beta$-D-Glc & $\begin{array}{c}\beta \text {-D-Xyl }(1 \longrightarrow 4)-\alpha \text {-L-Rha }(1 \longrightarrow 2)- \\
\alpha \text {-L-Ara }\end{array}$ & $\begin{array}{c}\mathrm{C}_{57} \mathrm{H}_{92} \mathrm{O}_{25} \\
1176\end{array}$ & {$[34]$} \\
\hline & $85^{*}$ & $\begin{array}{l}\alpha \text {-L-Ara }(1 \longrightarrow 2)-\beta \text {-D- } \\
\text { Glc }(1 \longrightarrow 2)-\beta \text {-D-Xyl }\end{array}$ & $\beta$-D-Glc & $\begin{array}{c}\mathrm{C}_{52} \mathrm{H}_{84} \mathrm{O}_{22} \\
1060\end{array}$ & {$[34]$} \\
\hline & $86^{*}$ & $\begin{array}{l}\beta-\mathrm{D}-\mathrm{Xyl}(1 \longrightarrow 2)-\beta-\mathrm{D}- \\
\mathrm{Glc}(1 \longrightarrow 2)-\beta-\mathrm{D}-\mathrm{Glc}\end{array}$ & $\beta$-D-Glc & $\begin{array}{c}\mathrm{C}_{53} \mathrm{H}_{86} \mathrm{O}_{23} \\
1090\end{array}$ & {$[34]$} \\
\hline & $87^{*}$ & $\begin{array}{l}\beta \text {-D-Glc }(1 \longrightarrow 2)-\beta \text {-D- } \\
\text { Glc }(1 \longrightarrow 2)-\alpha \text {-L-Ara }\end{array}$ & $\beta$-D-Glc & $\begin{array}{c}\mathrm{C}_{53} \mathrm{H}_{86} \mathrm{O}_{23} \\
1090\end{array}$ & {$[34]$} \\
\hline & $88^{*}$ & $\begin{array}{c}\alpha-\mathrm{L}-\mathrm{Ara}(1 \longrightarrow 2)-\beta-\mathrm{D}- \\
\text { Glc }(1 \longrightarrow 2)-\beta-\mathrm{D}-\mathrm{GluAME}\end{array}$ & $\beta$-D-Glc & $\begin{array}{c}\mathrm{C}_{54} \mathrm{H}_{86} \mathrm{O}_{24} \\
1118\end{array}$ & {$[35]$} \\
\hline & 89 & $\beta$-D-Glc $(1 \longrightarrow 2)-\alpha$-L-Ara & $\beta$-D-Glc & $\begin{array}{l}\mathrm{C}_{47} \mathrm{H}_{76} \mathrm{O}_{18} \\
928\end{array}$ & {$[34]$} \\
\hline \multirow{2}{*}{$\begin{array}{l}2 \beta, 3 \beta \text {-Dihydroxy-23-oxo- } \\
\text { olean-12en-28oic acid }\end{array}$} & 90 & $\beta$-D-GlcA & $\beta$-D-Glc & $\begin{array}{c}\mathrm{C}_{42} \mathrm{H}_{64} \mathrm{O}_{16} \\
824\end{array}$ & {$[32]$} \\
\hline & $91^{*}$ & $\begin{array}{l}\beta-\mathrm{D}-\mathrm{Xyl}(1 \longrightarrow 2)-\beta-\mathrm{D}- \\
\mathrm{Glc}(1 \longrightarrow 2)-\beta-\mathrm{D}-\mathrm{Glc}\end{array}$ & $\beta$-D-Glc & $\begin{array}{c}\mathrm{C}_{53} \mathrm{H}_{84} \mathrm{O}_{24} \\
1104\end{array}$ & {$[34]$} \\
\hline Soyasapogenol B & 92 & $\begin{array}{l}\alpha-\mathrm{L}-\mathrm{Rha}(1 \longrightarrow 2)-\beta-\mathrm{D}- \\
\mathrm{Gal}(1 \longrightarrow 2)-\beta-\mathrm{D}-\mathrm{GluA}\end{array}$ & - & $\begin{array}{c}\mathrm{C}_{48} \mathrm{H}_{78} \mathrm{O}_{18} \\
942\end{array}$ & {$[1,18,25,33,34]$} \\
\hline
\end{tabular}

Table 6: Saponins from M. arborea.

\begin{tabular}{|c|c|c|c|c|c|}
\hline Aglycone & No. & 3-OH substituted & 28-COOH substituted & Formula weight & Ref. \\
\hline \multirow{3}{*}{ Medicagenic acid } & 93 & $\beta$-D-GlcA & $\beta-\mathrm{D}-\mathrm{Xyl}(1 \longrightarrow 4)-\alpha-\mathrm{L}-\mathrm{Rha}(1 \longrightarrow 2)-\alpha$-L-Ara & $\mathrm{C}_{52} \mathrm{H}_{80} \mathrm{O}_{24} 1088$ & {$[1,25]$} \\
\hline & 94 & $\beta$-D-Glc & $\beta$-D-Xyl $(1 \longrightarrow 4)-\alpha-\mathrm{L}-\mathrm{Rha}(1 \longrightarrow 2)-\alpha$-L-Ara & $\mathrm{C}_{52} \mathrm{H}_{82} \mathrm{O}_{23} 1074$ & {$[1,25]$} \\
\hline & 95 & $\beta$-D-Glc & - & $\mathrm{C}_{36} \mathrm{H}_{56} \mathrm{O}_{11} 664$ & {$[1]$} \\
\hline
\end{tabular}

adipocytes [24]. Contemporary studies indicate that extracts rich in saponins are effective in lowering blood cholesterol levels. The potential beneficial effects of alfalfa saponins and flavonoids in agriculture and horticulture with regard to protecting plants against pests seem to be of great interest.

4.6. Saponins in Dietary Supplements. Various studies reported the use of alfalfa saponins in dietary supplements and are said to be linked with blood plasma parameters, nutrients digestibility, and growth performance of the cattle [48]. Medicago species mixed as hay and in silage are considered as significant food for herbivorous fauna, and a rich source of proteins and physically effective neutral detergent fiber for grazers [49]. Within natural grazing systems particularly in meadows, the intake of various classes of compounds like alkaloids, tannins, and saponins is being neutralized to give comfort to the grazers [50].

4.7. Bioavailability of the Saponins. The saponins have got permeability barrier across the cellular membranes for their large molecular weights. Hence the bioavailability of saponins should be checked as potential drugs. This major issue with larger molecular structures of saponins rendered them to catch the attention for utilization in drug industry. Recently, huge attempts were made to find the pharmacokinetics potential of these compounds (ginsenosides, astragaloside IV, clematichinenoside AR, and methylprotodioscin) sourced from different plants. In an attempt to find the reasons for the less permeability and reduced bioavailability of saponins, an in silico comparative study 
was done with crucial physicochemical parameters of cardiotonic drugs sourced from saponins/natural products to elucidate intestinal absorption and bioavailability [51].

\section{Conclusion}

The article summarizes the updates and latest advancements in various biological and pharmacological activities of structurally diverse saponins occurring in the genus Medicago. Medicago species ( $M$. sativa $L$.) are being used in traditional medicine systems due to the presence of unique saponins. The article produces the data of chemical structures and molecular masses of all saponins simultaneously. The biological activity of saponins is dependent on the number of side sugar chains attached to the sapogenins as well as to the nature of the sapogenin itself [52]. Monodesmosidic compounds were generally reported to be more biologically active than the corresponding bidesmosidic saponins [19]. For example, when pure aglycones have been used in in vitro bioassays, hederagenin was shown to be even more toxic than medicagenic acid and bayogenin against $\mathrm{X}$. index, while soyasaponin I, containing soyasapogenol B as a glycone, was the less-active saponin [53]. It is confirmed that structural diversity has a close relationship with its biological and/or pharmacological activities. It is suggested that more sophisticated techniques are needed to isolate more novel saponins for industrial, agricultural, and food manufacturing industries.

\section{Data Availability}

All data used to support the findings of this study are included within the paper.

\section{Conflicts of Interest}

The authors declare that there are no conflicts of interest regarding the publication of this paper.

\section{Acknowledgments}

This research was funded by the Key Lab of Natural Product Chemistry and Application at Universities of Education Department of Xinjiang Uygur Autonomous Region (2020YSHXZD02).

\section{References}

[1] K. A. Lesins and I. Lesins, "Genus Medicago (leguminosae). A taxogenetic study," Vegetatio, vol. 50, no. 2, p. 92, 1982.

[2] A. Tava and P. Avato, "Chemical and biological activity of triterpene saponins from Medicago species," Natural Product Communications, vol. 1, no. 12, 2006.

[3] A. Stochmal and W. Oleszek, "Seasonal and structural changes of flavones in alfalfa (Medicago sativa) aerial parts," Journal of Food, Agriculture and Environment, vol. 5, no. 2, p. 170, 2007.

[4] I. Kowalska, A. Stochmal, I. Kapusta et al., "Flavonoids from barrel medic (Medicago truncatula) aerial parts," Journal of Agricultural and Food Chemistry, vol. 55, no. 7, pp. 26452652, 2007.
[5] M. Jurzysta and G. R. Waller, "Antifungal and hemolytic activity of aerial parts of alfalfa (Medicago) species in relation to saponin composition. In: Saponins used in traditional and modern medicine," Advances in Experimental Medicine and Biology, vol. 404, pp. 565-574, 1996.

[6] P. Avato, D. Migoni, M. Argentieri, F. P. Fanizzi, and A. Tava, "Activity of saponins from Medicago species against HeLa and MCF-7 cell lines and their capacity to potentiate cisplatin effect," Anti-Cancer Agents in Medicinal Chemistry, vol. 17, no. 11, pp. 1508-1518, 2017.

[7] S. Sen, H. P. S. Makkar, and K. Becker, "Alfalfa saponins and their implication in animal nutrition," Journal of Agricultural and Food Chemistry, vol. 46, no. 1, pp. 131-140, 1998.

[8] P. T. Klita, G. W. Mathison, T. W. Fenton, and R. T. Hardin, "Effects of alfalfa root saponins on digestive function in sheep," Journal of Animal Science, vol. 74, no. 5, pp. 1144-1156, 1996.

[9] A. Kielbasa, A. Krakowska-Sieprawska, T. Kowalkowski, K. Rafinska, and B. Buszewski, "Distribution of sapogenins in morphological Medicago sativa L. parts: comparison of various extraction techniques," Journal of Separation Science, vol. 43, no. 3, pp. 671-680, 2020.

[10] B. Mickky, M. Abbas, and O. El-Shhaby, "Economic maximization of alfalfa antimicrobial efficacy using stressful factors," International Journal of Pharmacy and Pharmaceutical Sciences, vol. 8, no. 9, pp. 299-303, 2016.

[11] L. Adolfsson, H. Nziengui, I. N. Abreu et al., "Enhanced secondary- and hormone metabolism in leaves of arbuscular mycorrhizal Medicago truncatula," Plant Physiology, vol. 175, no. 1, pp. 392-411, 2017.

[12] M. Confalonieri, M. Cammareri, E. Biazzi et al., "Enhanced triterpene saponin biosynthesis and root nodulation in transgenic barrel medic (Medicago truncatulaGaertn.) expressing a novel $\beta$-amyrin synthase (AsOXA1) gene," Plant Biotechnology Journal, vol. 7, no. 2, pp. 172-182, 2009.

[13] V. Tzin, J. H. Snyder, D. S. Yang et al., "Integrated metabolomics Identifies CYP72A67 and CYP72A68 Oxidases in the biosynthesis of Medicago truncatula oleanate sapogenins," Metabolomics, vol. 15, no. 6, p. 85, 2019.

[14] A. Tava, M. Mella, P. Avato, M. P. Argentieri, Z. Bialy, and M. Jurzysta, "Triterpenoid glycosides from leaves ofMedicago arboreaL," Journal of Agricultural and Food Chemistry, vol. 53, no. 26, pp. 9954-9965, 2005.

[15] Z. Bialy, M. Jurzysta, M. Mella, and A. Tava, "Triterpene saponins from aerial parts ofMedicago arabicaL," Journal of Agricultural and Food Chemistry, vol. 52, no. 5, pp. 1095-1099, 2004.

[16] A. Saniewska, A. Jarecka, Z. Biały, and M. Jurzysta, "Antifungal activity of saponins originated from Medicago hybrida against some ornamental plant pathogens," Acta Agrobotanica, vol. 59, no. 2, pp. 51-58, 2006.

[17] T. D'Addabbo, P. Avato, and A. Tava, "Nematicidal potential of materials from Medicago spp," European Journal of Plant Pathology, vol. 125, no. 1, pp. 39-49, 2009.

[18] P. Abbruscato, S. Tosi, L. Crispino et al., "Triterpenoid glycosides from Medicago sativa as antifungal agents against pyricularia oryzae," Journal of Agricultural and Food Chemistry, vol. 62, no. 46, pp. 11030-11036, 2014.

[19] A. Tava and L. Pecetti, "Chemical investigation of saponins from twelve annual Medicago species and their bioassay with the brine shrimp Artemia salina," Natural Product Communications, vol. 7, no. 7, 2012. 
[20] C. Y. Cheok, H. A. K. Salman, and R. Sulaiman, "Extraction and quantification of saponins: a review," Food Research International, vol. 59, no. 5, pp. 16-40, 2014.

[21] A. Tava, C. Scotti, and P. Avato, "Biosynthesis of saponins in the genus Medicago," Phytochemistry Reviews, vol. 10, no. 4, pp. 459-469, 2011.

[22] A. Tava, M. Mella, P. Avato et al., "New triterpenic saponins from the aerial parts ofMedicago arabica(L.) huds," Journal of Agricultural and Food Chemistry, vol. 57, no. 7, pp. 28262835, 2009.

[23] Y. Fujii, S. Hirosue, T. Fujii, N. Matsumoto, H. Agematu, and A. Arisawa, "Hydroxylation of oleanolic acid to queretaroic acid by cytochrome P450 fromNonomuraea recticatena," Bioscience, Biotechnology, and Biochemistry, vol. 70, no. 9, pp. 2299-2302, 2006.

[24] A. Tava, M. Mella, Z. Bialy, and M. Jurzysta, "Stability of saponins in alcoholic solutions: ester formation as artifacts," Journal of Agricultural and Food Chemistry, vol. 51, no. 7, pp. 1797-1800, 2003.

[25] R. Montanari, D. Capelli, A. Tava et al., "Screening of saponins and sapogenins from Medicago species as potential PPAR $\gamma$ agonists and X-ray structure of the complex PPAR $\gamma /$ caulophyllogenin," Scientific Reports, vol. 6, no. 1, p. 27658, 2016.

[26] A. Tava, E. Biazzi, D. Ronga et al., "Triterpenic saponins from Medicago marina L," Phytochemistry, vol. 174, Article ID 112333, 2020.

[27] A. Tava, L. Pecetti, M. Romani, M. Mella, and P. Avato, "Triterpenoid glycosides from the leaves of two cultivars of Medicago polymorpha L," Journal of Agricultural and Food Chemistry, vol. 59, no. 11, pp. 6142-6149, 2011.

[28] P. Da Silva, V. Eyraud, M. Carre-Pierrat et al., "High toxicity and specificity of the saponin 3-GlcA-28-AraRhaxyl-medicagenate, from Medicago truncatula seeds, for Sitophilus oryzae," BMC Chemical Biology, vol. 12, no. 1, p. 3, 2012.

[29] Y. Ishiga, S. Rao Uppalapati, U. S. Gill, D. Huhman, Y. Tang, and K. S. Mysore, "Transcriptomic and metabolomic analyses identify a role for chlorophyll catabolism and phytoalexin during Medicago nonhost resistance against Asian soybean rust," Scientific Reports, vol. 5, no. 1, p. 13061, 2015.

[30] S. Goławska, "Deterrence and toxicity of plant saponins for the pea aphid acyrthosiphon pisum harris," Journal of Chemical Ecology, vol. 33, no. 8, pp. 1598-1606, 2007.

[31] S. Golawska, I. Lukasik, and B. Leszczynski, "Effect of alfalfa saponins and flavonoids on pea aphid," Entomologia Experimentalis et Applicata, vol. 128, no. 1, pp. 147-153, 2008.

[32] H. E. Witkowska, Z. Bialy, M. Jurzysta, and G. R. Waller, "Analysis of saponin mixtures from alfalfa (Medicago sativa L.) roots using mass spectrometry with MALDI techniques," Natural Product Communications, vol. 3, no. 9, 2008.

[33] H. Mazahery-Laghab, B. Yazdi-Samadi, M. Bagheri, and A. R. Bagheri, "Alfalfa (Medicago sativaL.) shoot saponins: identification and bio-activity by the assessment of aphid feeding," British Journal of Nutrition, vol. 105, no. 1, pp. 62-70, 2011.

[34] X.-G. Liu, Y.-Q. Sun, J. Bian et al., "Neuroprotective effects of triterpenoid saponins from Medicago sativa L. against $\mathrm{H} 2 \mathrm{O} 2$ induced oxidative stress in SH-SY5Y cells," Bioorganic Chemistry, vol. 83, pp. 468-476, 2019.

[35] D. Li, D. Liu, M. Lv, P. Gao, and X. Liu, "Isolation of triterpenoid saponins from Medicago sativa L. with neuroprotective activities," Bioorganic \& Medicinal Chemistry Letters, vol. 30, no. 4, Article ID 126956, 2020.

[36] C. Liu, C. M. Ha, and R. A. Dixon, "Functional genomics in the study of metabolic pathways in Medicago truncatula: an overview. In: functional genomics in Medicago truncatula," Methods in Molecular Biology, vol. 1822, pp. 315-337, 2018.

[37] T. D' addabbo, T. Carbonara, P. Leonetti, V. Radicci, A. Tava, and P. Avato, "Control of plant parasitic nematodes with active saponins and biomass from Medicago sativa," Phytochemistry Reviews, vol. 10, no. 4, pp. 503-519, 2011.

[38] Please provide complete publication details.

[39] M. M. Adel and E. A. Sammour, "Effect of sub-lethal dose of natural compound of Medicago sativa (L, Leguminaceae) on the hind gut and fat body of Spodoptera littoralis (Lepidoptera, Noctuidae)," Journal of Applied Sciences Research, vol. 8, no. 3, pp. 1398-1408, 2012.

[40] M. A. Ibrahim and H. A. Srour, "Saponins suppress nematode cholesterol biosynthesis and inhibit root knot nematode development in tomato seedlings," Natural Products Chemistry and Research, vol. 2, no. 1, pp. 1-6, 2013.

[41] M. Maestrini, A. Tava, S. Mancini, F. Salari, and S. Perrucci, "In vitro anthelmintic activity of saponins derived from Medicago spp. plants against donkey gastrointestinal nematodes," Veterinary Sciences, vol. 6, no. 2, p. 35, 2019.

[42] M. Maestrini, A. Tava, S. Mancini, D. Tedesco, and S. Perrucci, "In vitro anthelmintic activity of saponins from Medicago spp. against sheep gastrointestinal nematodes," Molecules, vol. 25, no. 2, p. 242, 2020.

[43] A. Balestrazzi, V. Agoni, A. Tava et al., "Cell death induction and nitric oxide biosynthesis in white poplar (Populus alba) suspension cultures exposed to alfalfa saponins," Physiologia Plantarum, vol. 141, no. 3, pp. 227-238, 2011.

[44] A. Krakowska, K. Rafińska, J. Walczak, T. Kowalkowski, and B. Buszewski, "Comparison of various extraction techniques of Medicago sativa: yield, antioxidant activity, and content of phytochemical constituents," Journal of AOAC International, vol. 100, no. 6, pp. 1681-1693, 2017.

[45] A. Kicel and M. A. Olszewska, "Evaluation of antioxidant activity, and quantitative estimation of flavonoids, saponins and phenols in crude extract and dry fractions of Medicago lupulina aerial parts," Natural Product Communications, vol. 10, no. 3, pp. 1934578X1501000-486, 2015.

[46] R. Gomathi, S. Banu, and K. Usha, "Phytochemical analysis and in vitro free radical scavenging activities of Medicago sativa seeds," Kongunadu Research Journal, vol. 2, no. 1, pp. 128-132, 2015.

[47] B. Sadowska, A. Budzyńska, M. Więckowska-Szakiel et al., "New pharmacological properties of Medicago sativa and Saponaria officinalis saponin-rich fractions addressed to Candida albicans," Journal of Medical Microbiology, vol. 63, no. 8, pp. 1076-1086, 2014.

[48] C. Liu, Y.-h. Qu, P.-t. Guo, C.-c. Xu, Y. Ma, and H.-l. Luo, "Effects of dietary supplementation with alfalfa (Medicago sativa L.) saponins on lamb growth performance, nutrient digestibility, and plasma parameters," Animal Feed Science and Technology, vol. 236, pp. 98-106, 2018.

[49] K. A. Beauchemin, "Effects of dietary neutral detergent fiber concentration and alfalfa hay quality on chewing, rumen function, and milk production of dairy cows," Journal of Dairy Science, vol. 74, no. 9, pp. 3140-3151, 1991.

[50] L. D. Lisonbee, J. J. Villalba, and F. D. Provenza, "Effects of tannin on selection by sheep of forages containing alkaloids, tannins and saponins," Journal of the Science of Food and Agriculture, vol. 89, no. 15, pp. 2668-2677, 2009.

[51] K. Yu, F. Chen, and C. Li, "Absorption, disposition, and pharmacokinetics of saponins from Chinese medicinal herbs: what do we know and what do we need to know more?" Current Drug Metabolism, vol. 13, no. 5, pp. 577-598, 2012. 
[52] T. D’Addabbo, M. P. Argentieri, J. Żuchowski et al., “Activity of saponins from Medicago species against phytoparasitic nematodes," Plants (Basel, Switzerland), vol. 9, no. 443, pp. 1-19, 2020.

[53] M. P. Argentieri, T. D’Addabbo, A. Tava, A. Agostinelli, M. Jurzysta, and P. Avato, "Evaluation of nematicidal properties of saponins from Medicago spp," European Journal of Plant Pathology, vol. 120, no. 2, pp. 189-197, 2008. 